\title{
Zur Entwicklung der Ringmuskelschicht an den Bronchien der Säugetiere.
}

\author{
Von
}

W. Kotzenberg, Würzburg.

Hierzu Tafel XXIII und 2 Textfiguren.

Seit langem sind an den primitiven Bronchien von Săugetierembryonen glatte Muskelfasern bekannt, welche dicht unter dem Epithel liegen, sehr frühzeitig auftreten und die einzige Hïlle der Bronchien bilden. Sie wurden von Kölliker (1) an Kaninchenembryonen und von Stieda (4) an Schafembryonen beschrieben.

Ersterer konnte sie an seinem Untersuchungsobjekt bereits am 14. Tage nachweisen, zu einer Zeit, wo wohl an der Speiseröhre, nicht aber an der Darmwand sich Muskelfasern schon vorfanden.

Kölliker sprach damals die Vermutung aus, dass diese Faserhaut möglicherweis e a us demEntodermenstanden sein könnte, da diese Zellen direkt dem Epithel angelagert erscheinen.

Es schien mir nun nicht unwert, diesem Gegenstand mit den Mitteln der modernen verbesserten Technik năher zu treten, um so mehr, als in einer kürzlich erschienenen Arbeit von Szili (5) die Frage der Unterscheidungsmöglichkeit zwischen epithelialen und mesodermalen glatten Muskelzellen erneut angeschnitten wurde, ja sogar die Vermutung ausgesprochen wurde, es sei vielleicht möglich, noch mancbe andere oder gar alle glatte Muskelelemente a uf epithelialen Ursprung zurückzuführen."

\section{Material und Methode.}

Die vorliegenden Untersuchungen wurden angestellt an Serien von Mäuseembryonen. Für die Wahl der Maus war ausschlaggebend, dass einerseits dieses Tier besonders grosse Elemente hat, andererseits die Beschaffung des Materials keine besonderen Schwierigkeiten bietet und die Serien nicht zu umfangreich wurden. 
Zur Entwicklung der Ringmuskelschicht an den Bronchien etc. $46 \mathrm{I}$

Die Embryonen wurden durchgehends in Zenker'scher Flüssigkeit konserviert, mit Boraxkarmin durchgefärbt und nach voraufgegangener Parafineinbettung in lückenlose Serien zerlegt. Vor der Einbettung wurden die Objekte in auffallendem Licht. bei $10-15$ facher Vergrösserung photographiert, und beziehen sich die Lăngenmaasse also auf die Grösse nach der Fixierung. Die Serien wurden nachträglich mit Haematoxylin. und Eosin gefärbt.

Die Durchfärbung mit Boraxkarmin bietet den Vorteil, dass. die Prăparate jederzeit später eine nachtrăgliche Kernfärbung mittelst einer beliebigen Farbe gestatten, während sie sonst nach Konservierung in Zenker- und anderen Chromsauregemischen nach kurzer Zeit schon ihre Färbbarkeit bekanntlich verlieren. Die Figuren wurden von dem Anatomiezeichner, Herrn Freitag, mit dem Abbé'schen Zeichenapparat entworfen.

Bevor ich zur Beschreibung der eigentlichen Untersuchung übergehe, möchte ich mir noch einige kurze Bemerkungen über die erste Anlage der Lunge überhaupt erlauben.

Willach (8) hat ziemlich eingehend die erste Anlage der Lunge bei Mäusen beschrieben. Seit dem Erscheinen dieser Abhandlung ist eine ganze Reihe von Arbeiten namentlich über die bereits vorher, besonders von A e by (9) und His (6) behandelte Frage: dichotomische oder monopodische Verzweigung der Bronchien erschienen, die eine ausführliche Zusammenstellungdurch L ühe (10) gefunden haben. Seitdem ist noch das grosse Werk von Narath (11) veröffentlicht worden, durch das mir diese Streitfrage wohl endgültig im Sinne der Dichotomie entschieden zu sein scheint ${ }^{1}$ ). Trotzdem möchte ich zur kurzen Orientierung Abbildungen zweier Lungenanlagen geben, wie ich sie aus Rekonstruktionen gewonnen habe, muss es mir aber versagen, auf die genannte Frage năher einzugehen, da das den Rahmen dieser Arbeit überschreiten würde.

Die erste Anlage der Lunge bei mus decumanus beschreibt Willach (8) in der Weise, dass zuerst an der Stelle, an welcher später die Lunge auftritt, sich eine Verdickung des Epithels des Vorderdarms findet, welche aus einer Vermehrung der Zellen dieser Schicht und Anordnung zu meheren Reihen bestand. Es

$\left.{ }^{1}\right)$ Die Arbeit war mir leider nicht in Original, sondern nur im Referate zugänglich. 
beginnt dann die Bildung der Lungenleiste (nach Kölliker), indem der ventrale Teil des Vorderdarmes sich vorwölbt. Im năchsten Stadium zeigte sich jederseits am unteren Ende dieser Lungenleiste eine taschenförmige Ausstülpung des Vorderdarmes die aber mit demselben in weit offner Verbindung standen."

Ich kann diese Angaben Willachs bestätigen und möchte noch einiges über die weitere Entwicklung (Stadien die Willach fehlten) hinzufügen.

In dem Stadium, welches die untenstehende Figur 1, welche aus einer Rekonstruktion ${ }^{1}$ ) auf Papier gewonnen wurde, darstellt, finden wir die oben beschriebenen "primăren Lungenbläschen“ bereits zu primăren Bronchialröhrchen ausgewachsen, welche auch auf dieser Entwicklungsstufe mit dem Vorderdarm noch in offner Verbindung stehen. Man sieht anf der Figur deutlich die bereits von Kölliker ( 3 a) und anderen beschriebene kolbenförmige Erweiterung des unteren Endes der Lungenleiste. Aus ihr wachsen die beiden primitiven Bronchialröhrchen hervor, welche an ihrer Abgangsstelle etwas verengt, an ibren Enden bläschenförmig erweitert erscheinen. Der rechte Bronchus ist dabei grösser und bereits besser ausgebildet als der linke, was auch Willach bereits bei seinen früheren Stadien konstatieren konnte.

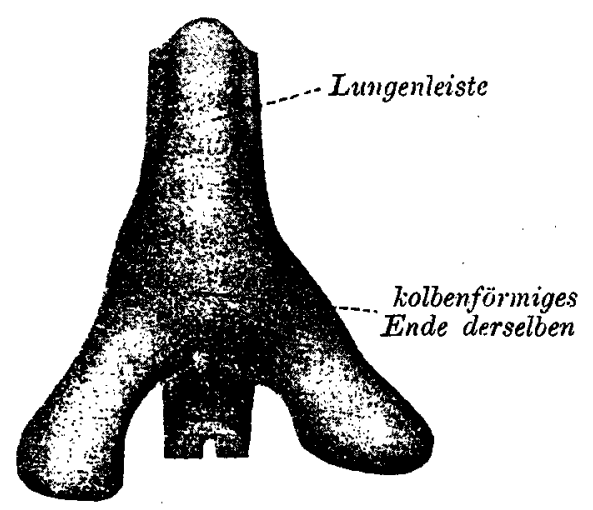

Figur 1.

Rekonstruktionsbild der Lungenanlage bei einem ca. $2 \mathrm{~mm}$ langen Mausembryo. Ventralansicht. Vergr. 150:1.

1) Die Richtigkeit der Rekonstruktion wurde später noch durch die B orn'sche Plattenmethode bestätigt. 
Zur Entwicklung der Ringmuskelschicht an den Bronchien etc. 463

Im mikroskopischen Bilde erscheint das Epithel der Lungenleiste als ein geschichtetes (inehrreihiges?), wăhrend das des dorsalen Teiles des Vorderdarmes einschichtig ist und kubische Zellen führt. In den Bronchialröhrchen jedoch ist bereits ein schönes hohes einreihiges Cylinderepithel entstanden. Eine Hülle der Bronchien ist noch nicht vorhanden; dieselben erscheinen vielmehr eingestülpt in das Speiseröhre und Lungenanlage gemeinsàm umgebende Mesoderm.

Im Verlauf der weiteren Entwicklung beginnt dann die Trennung der Trachea vom Oesophagus, indem die beiden Ränder der Verbindungsspalte miteinander verwachsen. Diese Trennung geht ziemlich langsam vor sich, so $z$ war, dass bei einem Embryo von $5 \mathrm{~mm}$ Länge die Lungenleiste sich immer noch auf 57 Schnitte bei $0,005 \mathrm{~mm}$ Schnittdicke, also über $0,28 \mathrm{~mm}$ erstreckte.

Die weitere Entwicklung zeigt Figur 2, in welcher die Verhältnisse der weiteren Bronchialverzweigung sichtbar sind und die ihrerseits für einen Beleg der dichotomischen Teilung gelten

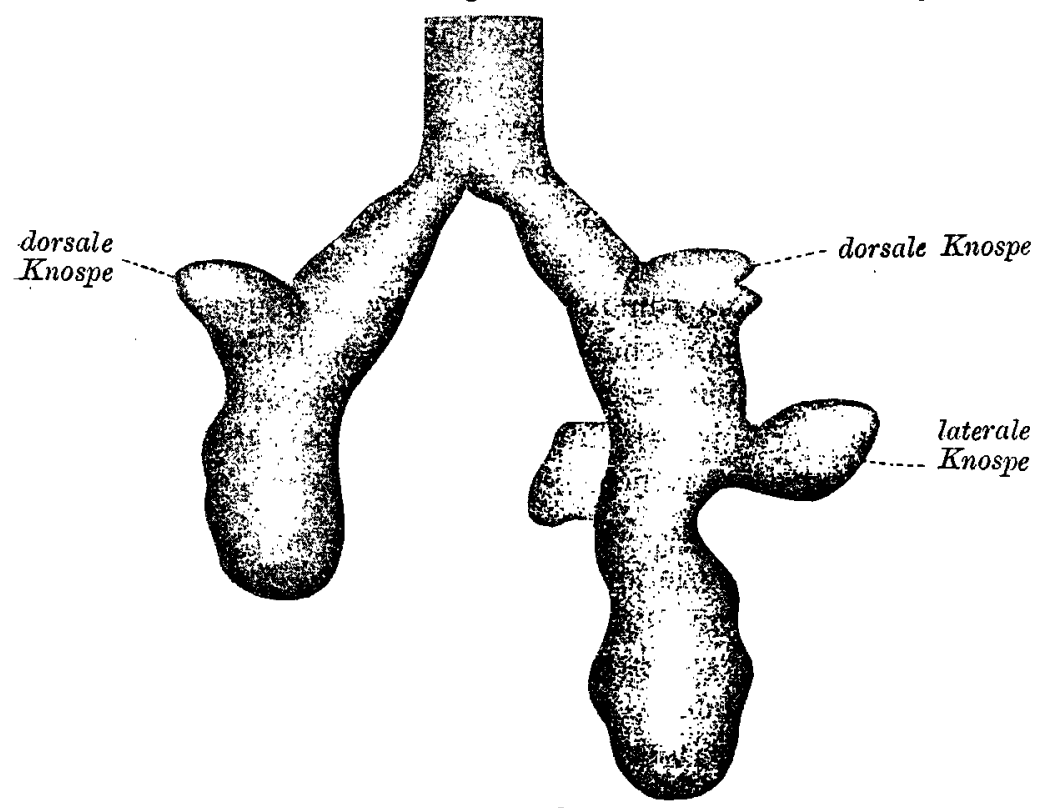

Figur 2.

Rekonstruktionsbild der Lungenanlage bei einem 6,4 mm langen Embryo. Dorsalansicht. Vergr. 100:1. 
kann, wenn man erstens die Anschwellungen beachtet, welche are den Abgangsstellen der Seitensprosse noch sichtbar sind und zweitens die Verånderung der Wachstumsrichtung des Stammbronchus nach dem jedesmaligen Abgang der Seitensprossen. Der rechte Bronchus zeigt je eine Knospe dorsal, lateral und ventral gerichtet, wahrend der linke erst eine dorsale Knospe erkennen lassst. Wir können auch hier wieder ein Ueberwiegen des rechten Bronchus über den linken konstatieren. Der weitere Ausbau der Lunge ist von verschiedenen Autoren [siehe $L$ üh e (10)] besonders von His (6) und Narath (11) für den Menschen und die Saugetiere zu genau beschrieben, um nochmals darauf eingehen zu können.

Gehen wir nun zur Betrachtung der mikroskopischen Befunde der eizelnen Serien über, so kann ich das in der Hauptsache bereits beschriebene erste Stadium übergehen, da es nichts Wesentliches zur Frage der Abstammung der Bronchialmuskulatur zeigt.

Das năchst ältere Stadium stammt von einem Embryo von $\mathrm{mm}$ Steiss-Nackenlănge; die Schnittdicke betrug $0,005 \mathrm{~mm}$. Figur 1 der Tafel stellt einen Querschnitt des linken Stammbronchus dar, von dem einen Teil in stärkerer Vergrösserung die Figur 2 wiedergibt.

Man sieht das Bronchialröhrchen durch eine scharfe Linie von dem umgebenden Mesenchym abgegrenzt. Das Röhrchen besteht aus einem einschichtigen Epithel, dessen hobe cylindrische Zellen offenbar in lebhafter Teilung begriffen sind. Die Kerne haben eine rundlich-ovale Form und liegen meist an der Basis der Zellen, scheinen jedoch beim Teilungsprozess an die Oberfläche des Lumens zu rücken, wie das ja auch von den Epithelzellen der Lieberkühn'chen Krypten bekannt ist. Das Protoplasma dieser Epithelzellen ist hell; ihre Lăngsaxe steht radiär zur Achse des Bronchus.

Man sieht im Epithel drei verschiedene Stadien der Zellteilung und zwar bei a) einen Mutterstern, bei b) Tochterstern und bei c) haben sich die Kerne bereits getrennt, liegen aber noch dicht beieinander. Es handelt sich dabei um eine Vermehrung der Epithelzellen selbst, die bedingt ist durch das starke Wachstum der Bronchien.

Würden diese Teilungsvorgange aber auf die Bildung einerausdem Epithel hervorgebenden Hülle 
Zur Entwicklung der Ringmuskelschicht an den Bronchien etc. 465

der Bronchien hindeuten, so müsste die Teilung in einer $z u$ der beschriebenen senkrechten Richtung erfolgen; und es müsste die beschriebene scharfe-Abgrenzung des Epithels an mindestens einer Stelle fehlen. Beides gelang in keinem Stadium der Entwicklung nachzuweisen.

Das Mesenchym ist vor allem ausgezeichnet durch eine grosse Anzahl von Kernteilungen, deren eine (bei $\mathrm{X}$ ) derart gegen die Wand des Bronchus andrăngt, dass es bei oberflächlicher Betrachtung aussieht, als entstamme sie dem Epithel. Figur 2 jedoch zeigt bei stärkerer Vergrösserung, dass sie thatsächlich dem Mesenchym angehört, indem die Epithelgrenze scharf im Bogen an $\mathrm{ihr}$ vorbeizieht. Im übrigen zeigen die Kerne des Mesenchyms in diesem Stadium noch kaum irgend welche Differenzierung ausser einigen, welche in der Nähe der eben beschriebenen Mitose liegen und eine längliche Gestalt aufweisen. (Vergl. Fig. 2).

Einen Umstand möchte ich noch nicht unerwähnt lassen, der jedoch in unserer Figur weniger hervortritt. Es ist das eine gewisse Neigung der dem Epithel zunächst gelegenen Kerne zu ringförmiger Anordnung um das Epithelrohr, während die weiter entfernten Kerne mehr ungeordnet durcheinander stehen. Es wird dies in Figur 3 einem um weniges alteren Stadium deutlicher zu Tage treten.

Figur 3 stammt ron einem Embryo von 6,4 mm Länge; die Schnittdicke betrug $0,01 \mathrm{~mm}$. Die Lungenanlage erstreckte sich über 62 Schnitte und weist rechts drei und links einen Seitenspross auf.

Am Oesophagus sind noch keine Muskelfasern differenziert Das Epithel des Bronchialröhrcbens ist ein hohes, einschichtiges aber mehrzeiliges Cylinderepithel. ${ }^{1}$ ) Auch hier scheinen die Kerne zur Teilung nach dem Lumen zu wandern. Die obere Mitose (bei $\mathrm{x}$ ) zeigt jedoch ein von dem oben beschriebenen abweichendes Verhalten, indem ihre Richtungsachse der Zellachse

1) Man erkennt hierbei den Effekt der in Fig. 1 beschriebenen starken Teilungsvorgänge im Epithel: Die Vergrösserung des Bronchus hat nicht Schritt gehalten mit der Vermehrung der Zellen, so dass diese sich übereinanderschieben. Weiter unten (in Fig. 5) werden wir das umgekehrte Verhalten bemerken.

Archir f. mikrosk. Anat. Bd. 60. 
parallel steht. Da ich jedoch weder in dieser noch in einer der. anderen Serien ein solches Verhalten ein zweites Mal gesehen habe, so glaube ich diesen Umstand auf eine Verschiebung. zurückführen zu dürfen, die mir durch das Vordrängen der der. Mitose nach links zunächst gelegenen Epithelzellen bedingt zu sein scheint.

Die Abgrenzung des Epithels ist auch in diesem Stadium wiederum eine absolut scharfe.

Dagegen zeigt das Mesenchym bereits eine deutliche Veranderung gegenüber der Figur 1. Die Zellen erscheinen nămlich ringförmig um das Epithelrohr herum verdichtet: das Protoplasma ist an dieser Zone dunkler, und die Kerne liegen mit ihren Achsen konzentrisch um das Röhrchen herum angeordnet. In diesem dunkleren Ring sowohl, wie in seiner nächsten Umgebung finden sich sehr zahlreiche Mitosen. Die Kerne des Mesenchyms selbst zeigen noch keine bestimmte Form, doch glaube ich, in der besprochenen Zone mehr Zellen von länglicher Gestalt erkennen zu können, während das übrige Mesenchym mehr runde Kerne aufweist.

Figur 4 stammt von einem Embryo von $8,7 \mathrm{~mm}$ SteissNackenlänge. Die Schnittdicke betrăgt $0,01 \mathrm{~mm}$. Die Lungenanlage erstreckte sich über 78 Schnitte. Am Oesophagus sind bereits glatte Muskelkerne zu erkennen, jedoch noch keine Differenzierung in Schichten. Am Darm sind Muskeln nicht nachzuweisen.

Das Epithelrohr besteht aus hohen, mehrreihigen Cylinderepithel- dessen Kerne teilweise mehr nach dem Lumen zugerückt sind und auch in diesem Stadium noch ziemlich lebhafte Kernteilungen zeigen, die a $\mathrm{ch}$ hier wieder in senkrechter Richtung zur Zellachse vor sich gehen. Die Abgrenzungslinie des Epithels gegen das Mesenchym ist auf allen Schnitten scharf und deutlich.

Das Mesenchym selbst besteht in grösserer Entfernung vom Epithel aus runden indifferenten Kernen; in der oben beschriebenen ringförmigen Verdichtungszone jedoch, das heisst in den dem Epithel zunachst liegenden Teilen des Mesenchyms sehen wir innerhalb eines Ringes von dunklerem Protoplasma nur noch vereinzelte runde Kerne liegen, wäbrend die meisten 
Zur Entwicklung der Ringmuskelschicht an den Bronchien efc. 467

Kerne eine schlanke, langlich ovale Form angenommen haben. Es kann nicht zweifélitit seîn, besonders wenn wir einen Vérgleich mit Figur ónstellen, dass wir es hier bereits mit den Vorlatern derglatten Muskelkerne zu thun haben.

In Figur jे haben wir einen Bronchus einer bereits gut entwickelten Lungenanlage vor uns. Dieselbe erstreckte sich bei einer Steiss-Nackenlänge des Embryo von 10,6 mm über eine Lange von $2,5 \mathrm{~mm}$. Der Oesophagus zeigte bereits deutích glatte Muskelfasern in schichtweiser Anordnung. Ebenso waren bereits am Darm glatte Muskeln zu erkennen.

Das Epithel des abgebildeten "n Bronchus bietet ungefăhr dieselben Verbăltnisse wie Figur 6 . Wir haben hohe cylindrische Zellen vor uns, deren Kerne jedoch nicht mehr in so lebhafter Teilung begriffen sind, wie in den beschriebenen Stadien. Infolgedessen drängen die Zellen nicht mehr übereinander: das Epithel beginnt den Charakter eines einfachen einreibigen anzunehmen. Gegen das Mesenchym ist das Epithelrohr wieder durch eine scharf contourierte Linie abgegrenzt.

Naturgemäss hat man in diesem Stadium, an dem der Bronchialbaum bereits zahlreiche Verzweigungen aufweisst, häufig. Schrägschnitte, auf denen dann diese Begrenzung verschwommen ist, doch wird man bei genauer Prüfung solche Stellen stets obne Weiteres erkennen and ausschalten können.

Dicht unter dem Fpithel findet sich ein Kranz von langen stäbchenförmigen Muskelkernen, welche mit ihrem dunkleren Protoplasma eine Hülle um das Bronchialrobr bilden. Auch die übrigen Zellen des Mesenchyms haben sith bereits soweit differenziert, dass um jeden Kern ein eigener Protoplasmahof sich gebildet hat.

\section{Zusammenfassung.}

Die Anlage der Lunge bei der Maus erfolgt bilateral durch Ausstülpung zweier Bläschen aus der rinnenförmig vorgebuchteten vorderen Schlundrohrwand. Frst wenn diese primären Lungenbläschen sich zu zwei primitiven Bronchien ausgewachsen haben, beginnt die Abschn̈urung der genannten Rinne vom Darmrohr und ihre Umwandlung in die Trachea. 
468 W. K o t z e n b e r g: Zur Entwicklung der Ringmuskelschicht etc.

Die Entwicklung der glatten Muskulatur der Bronchien geht sehr.frühzeitig vor sich aus den dem Epithelrobr zunachst gelegenen Kernen des Mesenchyms. Sie wird eingeleitet durch eine ringförmige Schichtung der Mesenchymkerne um das Röhrchen und das Auftreten zahlreicher Mitosen in dieser Zone.

Zum Schlusse gestatte ich mir, Herrn Professor Stö h r für die Anregung zu dieser Arbeit, sowie seine liebenswürdige Unterstützung bei Abfassung derselben meinen herzlichsten Dank zu sagen.

\section{Literaturverzeichnis.}

1. Kölliker: Hallenser Festschrift 1879, p. 119.

2. Derselbe: Zeitschrift für wissenseh. Zoologie. Bd. 40, p. 206.

3. Derselbe: Gewebelehre, 6. Aufl., p 138.

3a. Derselbe: Entwicklungsgesch. d. Menschen und der höheren Tiere.

4. Stied a: Zeitschrift für wissenschaftl. Zoologie, Bd. 30, Suppl. p. "111

5. Szili: Zur Anatomie und Entwicklungsgeschichte der hinteren Irisschichten mit bes. Berücksichtigung des sphincter irid. des Menschen. Anat Anzeig. XX, Heft 7.

6. His: Archiv f. Anatomie and Entwicklungsgeschichte, 1887, p. 90.

7. O. Hertwig: Lebrbuch der Entwicklungsgeschichte des Menschen und der Wirbeltiere.

8. Willach: Beiträge zur Entwicklnng der Lunge bei Säugetieren. Osterwieck a. H., 1888.

9. A e by: Der Bronchialbanm d. Säugetjere und des Menschen. Leipzig 1880.

10. Lü h e: Der Bronchialbaum der Säugetiere, Zool. Zentralblatt, 1901, 34.

11. Narath: Der Bronchialbaum der Sängetiere und des Menschen. Bibliotheca medica, Abt. A, Anatomie, 1901, Heft 3.

\section{Figurenerklärung der Tafel XXIII.}

Figur 1: Querschnitt durch den linken Stammbronchus eines 8;7 mm langen Mausembryo von $5 \mathrm{~mm}$ Länge. Vergr. 400.

Figur 2: Teil des Querschnitts von Fig 1. Vergr. 850.

Figur 3: Querschnitt durch den linken Stammbronchus eines 6,4 $\mathrm{mm}$ langen Mausembryo. Vergr. 400.

Figur 4: Querschnitt durch den rechten Hauptbronchus eines $8,7 \mathrm{~mm}$ langen Mausembryo. Vergr. 700.

Figur 5: Querschnitt durch ein Bronchialröhrchen eines $10,6 \mathrm{~mm}$ langen Mausembryo. Vergr. 900. 